\title{
A general-purpose data extraction language
}

\author{
WAI HON TSUI and PETER VAN GELDER \\ Psychiatry Department, New York University Medical Center, New York, New York 10016
}

\begin{abstract}
A large and diverse set of experiments is run by a single data acquisition program. The resulting single-output file format encompassing all possible experimental paradigms allows a corresponding single program to be written to extract data from these files to accumulate descriptive statistics. However, this diversity requires extreme flexibility in specifying the classes of data to be extracted, so that, for example, any sort of sequential effects can be observed in reaction times. An additional constraint is to remove all program complexity other than that imposed by the analysis logic itself, so that psychologists can program their own data analyses. The resulting analysis program provides a general-purpose data extraction language with which the user specifies the desired classes of data to be formed into lists for further operations by the program. The language structure incorporates the natural hierarchy of the data base structure itself and provides a record-specifier syntax with which the class of records at each level of the hierarchy may be selected. Arithmetic, comparison, logical, statistical, and list processing functions are provided that allow array arguments to produce arrays of functional evaluations. These functions can be nested and otherwise combined into expressions to further define classes of data.
\end{abstract}

The information processing laboratory of the psychiatry department, New York University Medical Center, has undertaken to run a diverse set of perceptual experiments, each with various subtasks, all designed to maximize the amount of information obtained from the subject. A major goal of the data analysis software is to facilitate the extraction of as many aspects of this information as seem relevant to the study of attention.

The experimental examples given by Van Gelder, Todd, and Tsui (1979) can serve as prototypes for illustrative data analyses. For the Stroop color-word task, the preliminary conditions generate sequential effects to show the effect of the preceding time interval or preceding stimulus sequence, respectively, on the current response. Main effects establish individual response baselines for each of the four response keys. In the interference condition, main effects are important, and sequential effects due to runs of similar stimulus classes serve to refine this information.

The continuous performance test has traditionally been used to tally errors of omission and commission for this vigilance task. Our implementation provides reaction time measures, as well as error probabilities. We change the target stimulus assignment every three lists, so all summary statistics are provided for each half-list to show the contrasting effects of switching and vigilance. In the speeded version, where occurrences of the target letter are counted during a list and reported afterward, the magnitudes of response errors are tallied as functions of the correct count, list number, and target prevalence early vs. late in the list. Second-guess

Send correspondence to Peter Van Gelder, Psychiatry Department, New York University Medical Center, 550 First Avenue, New York, New York 10016. error magnitudes are tallied as functions of firsterror magnitudes and list number.

The pursuit eye-tracking task provides a wealth of information in the response waveform. An EOG analysis program has been written to extract discrete oculomotor events from the digitized analog data. Then, these events are analyzed as discrete data to provide distributions of saccades, velocity arrests, fixations, and blinks in the pursuit tracking cycle.

Our intent was to enable a user to extract measures of the data easily and interactively. One envisions a sort of data-base interrogation system, where an experimenter conceives a question to ask of the data, rushes to the terminal, and produces the answer. The only limitation to such an exchange should be the complexity of the question itself, which in a general-purpose data extraction system may take some thought to produce the required clarity of expression. But all programming constructs should be optional, so that a simple question can be asked simply and a complex question can be asked powerfully.

The analysis sytem must also lend itself to batch runs of many measures. Our application involves a cluster analysis phase, in which hundreds of these measures are combined in various ways in an effort to identify components of attention. Measures can be developed interactively using pilot data and saved for later batch rerun with the complete data set.

These analysis requirements can, at best, be met with a single discrete analysis program that accesses a well structured, uniformly specified data base. The required uniform data file format has been insured in our system by the use of a single data acquisition program. The resulting analysis program provides a general-purpose data extraction language with which the user specifies 
the desired classes of data to be formed into lists for further operations by the program. The language structure incorporates the natural hierarchy of the data-base structure itself and provides a record-specifier syntax with which the class of records at each level of the hierarchy may be selected.

\section{STRUCTURE OF THE DATA BASE}

The data collected in one experimental session are output to a file that is identifiable by a unique usersupplied file name (ID). An experimenter organizes his experiment into blocks of stimulus-response events. Accordingly, a file is organized into blocks of events. The data for each event include (1) a description of the stimuli presented to the subject, in terms of the name and the position (i.e., the starting $\mathrm{X}$ - and $\mathrm{Y}$-coordinates on the screen) of each stimulus, (2) the correct response (RC) expected from the subject, (3) the actual response (RA) received from the subject, (4) the subject's reaction time ( $R T$ ), and (5) the duration (TIME) of each variable wait period in the event.

Such data organization fits very well into a hierarchical structure. A file, a block, an event, a stimulus, and a period are records at different levels of the hierarchy. A block is said to be a subrecord of a file. An event is a subrecord of a block. A stimulus and a period are subrecords of an event. Besides subrecords, there are also data fields associated with each record. For example, ID is a field of a file and RT is a field of an event. In each of these fields, a primitive datum is stored, which can either be a number or a character string. Figure 1 illustrates the organization of such a hierarchy.

\section{DATA EXTRACTION}

Organizing data in a hierarchical structure like the one just described enables the extraction of data of dissimilar natures in a uniform manner: The user selects the appropriate records first and then specifies the kind of data desired from the records.

The mechanism used to select records is based on this hierarchy of records. For example, the selection of

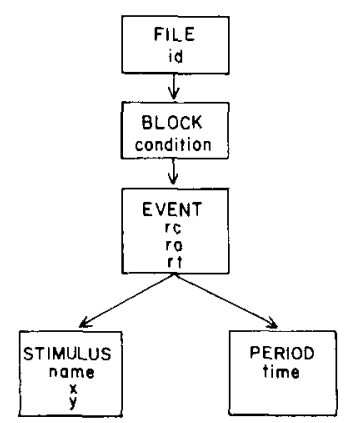

Figure 1. The hierarchical organization of the data base. the first event of the second block in the fifth file is specified by

\section{FILE [5] BLOCK [2] EVENT [1]}

This notation can easily be generalized to allow the selection of any number of records. For example, the selection of the first 30 events of the second block in the fifth file is specified by

\section{FILE [5] BLOCK [2] EVENT [1,30]}

and the selection of all events of the second block in the fifth file is specified by

\section{FILE [5] BLOCK [2] EVENT}

In general, each of these sentences selects a list of records. Hence, they are called record lists.

The only criterion used by this scheme to select records is their natural ordering. However, this selection criterion is not sufficient in most cases. For instance, we might want to look at those and only those events in which a certain stimulus is presented, or ignore those blocks with too many early responses, or examine only those events in which the subject responded correctly. To be able to specify the select criteria for these diversified situations, a more sophisticated scheme is needed, like the predicate calculus used by logicians. For example, the selection from the first 30 events of the second block in the fifth file of all those events in which the subject responded correctly is specified by

\section{FILE [5] BLOCK [2] EVENT [1, 30] : (EQ RC RA)}

where the predicate (EQ RC RA), written in Polish notation, is true if the correct response (RC) equals the actual response (RA). A battery of functions is provided for the construction of these predicates. This will be discussed more thoroughly in the next section. In general, the record specifier for each record level of the hierarchy may be indexed (up to three numbers or expressions, enclosed in square brackets, indicating first and last record number and record number increment, respectively, as in a FORTRAN DO loop) and qualified, by including only those records for which the expression following the colon evaluates as true. These expressions are built from logical or comparison functions, any of whose arguments can themselves be functions.

Once record selection is made, the next step is to specify what to do with the record selected. There are two ways that a record list can be used: list expressions and statement groups.

\section{List Expressions}

In a list expression, a list of records is first selected by a record list and then the kind of data that should 
be extracted from these records is selected by an expression. The result is either a list of numbers or a list of character strings, depending, of course, on the type of data extracted. The format

$$
\text { record list expression }
$$

defines a list expression. In general, expression can be any acceptable data description, but it is often just the name of a field in the selected list of records. For example, the list expression

\section{FILE: (EQ ID 'ONSAM34') BLOCK [2] EVENT [1, 30] RT}

denotes a list of reaction times for the first 30 events of the second block in the file whose file name is ONSAM34.

\section{Statement Groups}

A statement group is a looping facility that allows each record selected by a record list to be examined in greater detail. It has the format

BEGIN (record list)
statement $_{1}$
statement $_{2}$
$\cdot$
$\cdot$
statement
END

The statements in a statement group specify precisely which action is to be taken on each of the records selected by the record list. For example, the statement group

\section{BEGIN (FILE [5] BLOCK) TYPE CONDITION END TYPE MEAN EVENT: (EQ RC RA) RT}

looks at all the blocks in the fifth file and, for each of these blocks, it types on the terminal the experimental condition name of the block and the mean reaction time over all the events of the block in which the subject responded correctly. This example also illustrates how a list expression can be used. The list expression "EVENT: (EQ RC RA) RT" in the second statement forms a list of reaction times for all the events (in the current block) that satisfy the predicate (EQ RC RA), the function MEAN is then applied to this list of reaction times to obtain the mean value, which is subsequently typed on the terminal.

\section{FUNCTIONS}

An application of a function is written in the form ( $f x_{1} x_{2} \ldots x_{n}$ ), where $f$ is the name of the function and $x_{1}, x_{2}, \ldots, x_{n}$ are the arguments. The parentheses around this expression are entirely optional, but they do improve readability in many cases.

In an example above, the function EQ is used in the predicate (EQ RC RA) to determine the equality of the $\mathrm{RC}$ and RA fields in an event. This use of a function enables the selection of records on a conditional basis. In another example, the function MEAN is used to evaluate the mean value of a list of reaction times. This use of a function enables us to evaluate data after they have been extracted.

Many other functions are also provided. Some perform comparison operations: EQ, NE (not equal), GT (greater than), and LT (less than). Some perform logical operations: AND, OR, and NOT. Some perform arithmetical operations: ADD, SUB, MUL, DIV, and EXP (exponentiation). Some perform statistical operations: COUNT, MEAN, SD, TALLY (for N, sum, and sum of squares), PER (percentile), and MEDIAN. Finally, some functions perform list and string operations: CON (concentration of lists), EXT (extraction of an element from a list), CHAR (extraction of a character from a character string), and MATCH (template matching on strings). Among these functions, the reader might find MATCH most unfamiliar. It matches a character string (the second argument) against a template (the first argument). The template specification uses three special constructions. A list of character strings separated by commas and enclosed in brackets allows any of the specified strings in the bracketed position in the template. An asterisk allows any single character in that position in the template. A dash allows any character string in that position in the template. The last two constructions follow Data General RDOS conventions. For example, the predicate (MATCH '*[A,D]-' NAME) is true if the second character of the name of a stimulus is $\mathrm{A}$ or $\mathrm{D}$. This function is very useful for the classification of stimuli, experimental conditions, and data files based on the attributes encoded in their names.

\section{STATEMENTS}

Statements that perform assignment, looping, and conditional branching are provided. An assignment is written in the form $v=e$. It assigns the value of an expression e to the variable $v$. The e can be any type of expression: a list expression, an application of a function, a reference to another variable, and so on.

We have already seen one type of loop facility in the form of a statement group that iterates over a list 
of records. Conventional looping is also provided, but in a format much closer to that of a statement group:

BEGIN (v[i,j,k])
statement
statement
$\cdot$
$\cdot$
$\cdot$
statement
END

This loop executes statement $t_{1}$ through statement $t_{n}$ sequentially for each value of the variable $\mathrm{v}$ as specified by the three parameters $i, j$, and $k$ ( $i$ is the starting value of $v, j$ if the final value of $v$, and $k$ is the value that $v$ is to be incremented at the end of each iteration).

Conditional branching is provided in the form of an if statement:

\section{IF e THEN statement ${ }_{1}$ ELSE statement ${ }_{2}$}

where statement $t_{1}$ is executed if the expression e is true and statement ${ }_{2}$ is executed otherwise. The last part, "ELSE statement ${ }_{2}$," can be omitted, in which case, no operation will be performed if $e$ is false.

\section{ARRAYS}

An array is simply a sequence of data elements. An array of $n$ elements is formed by writing $<e_{1}, e_{2}, \ldots$, $e_{n}>$, when $e_{i}$ is an expression whose value is going to be the ith element of the array. Suppose this array is assigned to the variable $A$. Then its ith element is referenced by the expression $\mathrm{A}[\mathrm{i}]$. Its jth element can also be changed to the value of an expression e by an assignment statement of the form $\mathrm{A}[\mathrm{j}]=\mathrm{e}$.

In data extraction, arrays are primarily used for the classification of data. An array of predicates can be used to specify separate classes of criteria for record selection. The following statement is a typical application of this concept:

A $=$ EVENT $:<$ predicate $_{1}$, predicate $_{2}, \ldots$, predicate $_{n}>$ expression

It has the same effect as the $\mathrm{n}$ statements below:

$\mathrm{A}[1]=$ EVENT $:$ predicate $_{1}$ expression

$\mathrm{A}[2]=$ EVENT $:$ predicate $_{2}$ expression

$\mathrm{A}[\mathrm{n}]=$ EVENT $:$ predicate $_{\mathrm{n}}$ expression

For example, A = EVENT: $\angle$ EQ RC RA, NE RC RA $>$ RT assigns an array of two elements to the variable $A$. The first element is a list of reaction times for all the events in which the subject responded correctly, and the second element is a list of reaction times for all the events in which the subject responded incorrectly.

To fully utilize this classification capability, the notion of a function is generalized by allowing arrays to be its arguments. For a function $f$ with one argument, $\mathrm{f}\left\langle\mathrm{n}_{1}, \mathrm{n}_{2}, \ldots, \mathrm{n}_{\mathrm{k}}>\right.$ is defined to be the array

$$
<\mathrm{f} \mathrm{n}_{1}, \mathrm{f}_{2}, \ldots, \mathrm{f} \mathrm{n}_{\mathrm{k}}>\text {. }
$$

For a function $\mathrm{f}$ with two arguments, $\mathrm{f}<\mathrm{m}_{1}, \mathrm{~m}_{2}, \ldots$, $m_{k}>\left\langle n_{1}, n_{2}, \ldots, n_{\ell}\right\rangle$ is defined to be the array (of size $k \times l$ )

$$
\begin{gathered}
<f m_{1} n_{1}, f m_{1} n_{2}, \ldots, f m_{1} n_{\ell} \\
f m_{2} n_{1}, f m_{2} n_{2}, \ldots, f m_{2} n_{\ell}, \\
\cdot \\
\cdot \\
f m_{k} n_{1}, f m_{k} n_{2}, \ldots, f m_{k} n_{\ell}>.
\end{gathered}
$$

For example, the result of $\mathrm{ADD}<10,20\rangle<1,2,3\rangle$ is the array

$$
\langle 11,12,13,21,22,23\rangle \text {. }
$$

This use of this facility is demonstrated in the following statement: RTS = MEAN EVENT: AND (EQ NAME <'A', 'B', 'C'>) <EQ RC RA, NE RC RA> RT. This statement classifies events into six categories, depending whether the stimulus presented to the subject is called $\mathrm{A}, \mathrm{B}$, or $\mathrm{C}$, and whether the subject responded correctly or not. The value assigned to RTS is an array whose elements are each the mean reaction time for one of these six classes of events.

\section{AN EXAMPLE}

We now consider the cued choice reaction time experiment described by Van Gelder et al. (1979). In that experiment, a cue and a choice stimulus, both chosen from a set of four stimuli, are presented to the subject in each trial. Two stimuli of this set are auditory and two are visual. These stimuli are mapped onto two responses, with one visual and one auditory stimulus assigned to each response. The names of the four stimuli are $A L, A R, V L$, and VR; the first letter represents the mode $(A=$ auditory, $V=$ visual $)$, and the second letter represents the response hand assigned to the stimulus ( $\mathrm{L}=$ left, $\mathrm{R}=$ right). By embedding attributes of stimuli in their names, we simplify data analysis enormously. For example, Figure 2 is a program, written in the data extraction language, that allows us to study the cuing and sequential effects in this particular experiment.

This program examines each block separately (Line 1). The first statement on Line 2 prints three 


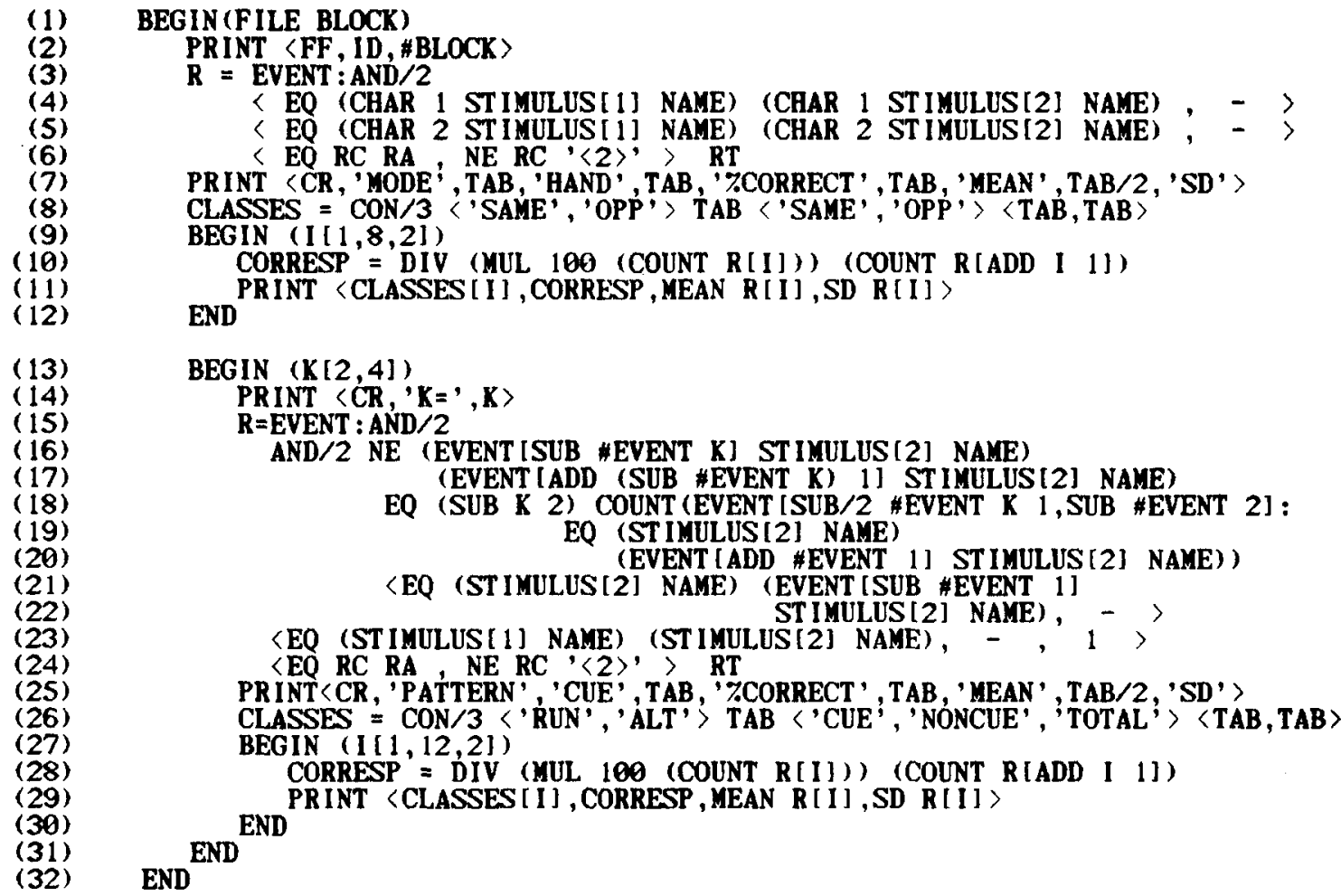

Figure 2. A program written to extract data on cuing and sequential effects for a choice reaction time experiment.

items: a form feed (so that data for each block are printed on a new page), the name of the data file, and the block number (\#BLOCK). The cuing effects are studied first (Lines 3-12).

The assignment statement on Lines 3-6 builds an array of eight lists to classify events into eight categories with respect to three dimensions: modality (cued vs. noncued, as on Line 4), response hand (cued vs. noncued, as on Line 5), and subject's response (correct responses vs. all responses except for catch trials, as on Line 6). Note that in this statement (1) AND/2 represents the two occurrences of the word AND, hence the record list "EVENT: AND/2 $e_{1} e_{2} e_{3}$ " selects events that satisfy all three logical expressions $e_{1}, e_{2}$, and $e_{3}$. (2) The dash in an array of logical expressions represents the negation of the preceding logical expression. (3) The function CHAR extracts a character from a character string; in particular, the results of (CHAR i s) is the ith character of the character string s. (4) In this experiment, a noncatch trial event can be determined by the logical expression (NE RC ' $<2>$ '), as on Line 6.

Although the events are classified into eight categories, reaction time data are computed only for four of them. The other four categories, corresponding to (NE RC ' $<2>$ '), are here to facilitate the computation of percent correct response (Line 10) with respect to the four cue conditions. The data for cuing effects are tabulated in a 4 by 5 table (see Figure 3). The four rows correspond to the four cue conditions: (1) both modality and hand are cued, (2) modality is cued but hand is not, (3) hand is cued but modality is not, and (4) neither is cued. The five columns contain data for each of the four cue conditions. The first two (constructed on Line 8 ) are mode and hand specifying the corresponding cue condition, followed by the percent correct response and the mean and standard deviation of correct reaction times for that particular cue condition (Line 11). The heading for the table is printed as on

\begin{tabular}{|c|c|c|c|c|c|c|}
\hline \multicolumn{2}{|c|}{ 149VAOBRH2 } & \multicolumn{5}{|l|}{1} \\
\hline $\begin{array}{l}\text { YODE } \\
\text { SAME } \\
\text { SAME } \\
\text { OPP } \\
\text { OPP }\end{array}$ & $\begin{array}{l}\text { HAND } \\
\text { SAME } \\
\text { OPP } \\
\text { SAME } \\
\text { OPP }\end{array}$ & $\begin{array}{c}\text { 7CORRECT } \\
91 \\
100 \\
109 \\
33\end{array}$ & $\begin{array}{l}\quad \text { MEAN } \\
0.31723 \mathrm{E} \\
0.39450 \mathrm{E} \\
0.54333 \mathrm{E} \\
0.48800 \mathrm{E}\end{array}$ & $\begin{array}{l}3 \\
3 \\
3 \\
3\end{array}$ & $\begin{array}{c}\mathrm{SD} \\
0.68266 \mathrm{E} \\
0.82500 \mathrm{E} \\
0.56958 \mathrm{E} \\
0.00000 \mathrm{E}\end{array}$ & $\begin{array}{l}2 \\
2 \\
2 \\
0\end{array}$ \\
\hline$K=$ & 2 & & & & & \\
\hline $\begin{array}{l}\text { PATTERN } \\
\text { RUN } \\
\text { RUN } \\
\text { RUN } \\
\text { ALT } \\
\text { ALT } \\
\text { ALT }\end{array}$ & $\begin{array}{l}\text { CUE } \\
\text { CUE } \\
\text { NONCUE } \\
\text { TOTAL } \\
\text { CUE } \\
\text { NONCUE } \\
\text { TOTAL }\end{array}$ & $\begin{array}{c}\text { 7CORRECT } \\
100 \\
100 \\
100 \\
87 \\
66 \\
81\end{array}$ & $\begin{array}{l}\text { MEAN } \\
0.30550 \mathrm{E} \\
0.48900 \mathrm{E} \\
0.36667 \mathrm{E} \\
0.32964 \mathrm{E} \\
0.48250 \mathrm{E} \\
0.3636 \mathrm{IE}\end{array}$ & $\begin{array}{l}3 \\
3 \\
3 \\
3 \\
3 \\
3\end{array}$ & $\begin{array}{c}\text { SD } \\
0.15000 \mathrm{E} \\
0.00000 \mathrm{E} \\
0.86511 \mathrm{E} \\
0.79833 \mathrm{E} \\
0.11168 \mathrm{E} \\
0.10848 \mathrm{E}\end{array}$ & $\begin{array}{l}1 \\
0 \\
2 \\
2 \\
3 \\
3\end{array}$ \\
\hline$K=$ & 3 & & & & & \\
\hline $\begin{array}{l}\text { PATTERN } \\
\text { RUN } \\
\text { RUN } \\
\text { RUN } \\
\text { ALT } \\
\text { ALT } \\
\text { ALT }\end{array}$ & $\begin{array}{l}\text { CUE } \\
\text { CUE } \\
\text { NONCUE } \\
\text { TOTAL } \\
\text { CUE } \\
\text { NONCUE } \\
\text { TOTAL }\end{array}$ & $\begin{array}{c}\text { 7CORRECT } \\
100 \\
0 \\
100 \\
100 \\
50 \\
75\end{array}$ & $\begin{array}{l}\text { MEAN } \\
0.31300 \mathrm{E} \\
0.00000 \mathrm{E} \\
0.31300 \mathrm{E} \\
0.32300 \mathrm{E} \\
0.48800 \mathrm{E} \\
0.37800 \mathrm{E}\end{array}$ & $\begin{array}{l}3 \\
0 \\
3 \\
3 \\
3 \\
3\end{array}$ & $\begin{array}{c}\text { SD } \\
0.60000 \mathrm{E} \\
0.00000 \mathrm{E} \\
0.00000 \mathrm{E} \\
0.39000 \mathrm{E} \\
0.60000 \mathrm{E} \\
0.84048 \mathrm{E}\end{array}$ & $\begin{array}{l}0 \\
0 \\
0 \\
2 \\
6 \\
2\end{array}$ \\
\hline$K=$ & 4 & & & & & \\
\hline $\begin{array}{l}\text { PATTERN } \\
\text { RUN } \\
\text { RUN } \\
\text { RUN } \\
\text { ALT } \\
\text { ALT } \\
\text { ALT }\end{array}$ & $\begin{array}{l}\text { CUE } \\
\text { CUE } \\
\text { NONCLE } \\
\text { TOTAL } \\
\text { CUE } \\
\text { NONCUE } \\
\text { TOTAL }\end{array}$ & $\begin{array}{c}\text { ZCORRECT } \\
0 \\
\theta \\
0 \\
100 \\
0 \\
100\end{array}$ & $\begin{array}{l}\text { MEAN } \\
0.00000 \mathrm{E} \\
0.00000 \mathrm{E} \\
0.00000 \mathrm{E} \\
0.32200 \mathrm{E} \\
0.00000 \mathrm{E} \\
0.32200 \mathrm{E}\end{array}$ & $\begin{array}{l}0 \\
0 \\
0 \\
3 \\
0 \\
3\end{array}$ & $\begin{array}{c}\text { SD } \\
0.00000 \mathrm{E} \\
0.00000 \mathrm{E} \\
0.00000 \mathrm{E} \\
0.00000 \mathrm{E} \\
0.06000 \mathrm{E} \\
0.00000 \mathrm{E}\end{array}$ & $\begin{array}{l}0 \\
0 \\
0 \\
0 \\
0 \\
0\end{array}$ \\
\hline
\end{tabular}

Figure 3. Sample data for one block, generated by the analysis program of Figure 2. 
Line 7, and then each row of the table is printed by the loop on Lines 9-12.

The last half of the program (Lines 13-31) examines the sequential effects and their relation to cuing effects. We are interested in two types of patterns of events, runs vs. alternations. A run of length $K$ means a sequence of exactly $K$ consecutive events with the same choice stimulus. An alternation of length $\mathrm{K}$ means a sequence of exactly $K-1$ consecutive events with the same choice stimulus followed by an event with a different choice stimulus. The effects of runs and alternations are studied for $\mathrm{K}=2,3$, and 4 (Line 13).

First, the events are classified into 12 categories with respect to three dimensions: pattern type (run vs. alternation, as on Lines 16-22), cue condition (cued vs. noncued vs. don't-care, as on Line 23), and subject's response (correct response vs. all responses, as on Line 24). An event belongs to a run (respectively alternation) of length $\mathrm{K}$ if all of these three conditions are satisfied (let $i$ denote the number of that event): (1) The choice stimuli for the $\mathrm{i}-\mathrm{k}$ th event and the $\mathrm{i}-\mathrm{k}+1$ th event are different (Lines 16-17). (2) The choice stimuli for the $\mathrm{i}-\mathrm{k}+1$ th, $\mathrm{i}-\mathrm{k}+2$ th, . . . and $\mathrm{i}-1$ th events are all the same (Lines 18-20). (3) The choice stimuli for the $\mathrm{i}-1$ th and the ith events are the same (respectively different) (Lines 21-22). The 12 categories of events are mapped onto six different conditions (two pattern types vs. three cue conditions). The data for each of the six conditions, including the percentage and the mean and standard deviation of reaction times of correct responses, are printed (Lines 25-32) in the form of a 6 by 5 table similar to the one used on cuing effects (see Figure 3).

\section{REFERENCE}

Van Gelder, P., Todd, J., \& Tsui, W. H. Data acquisition software for high data rate experiments. Behavior Research Methods \& Instrumentation, 1979, 11, 192-198. 